\title{
Efecto anticonceptivo y postcoital del extracto etanólico de lashojas del Desmodium molliculum (HBK).DC "Manayupa" en ratas hembras Holtzmann
}

\section{Postcoital contraceptive effect of ethanolic extract of the leaves of Desmodium molliculum (HBK).DC "Manayupa" in Holtzmann female rats}

\author{
Fidel Ernesto Acaro Chuquicaña
}

Facultad de Medicina, Universidad Nacional Mayor de San Marcos

\begin{abstract}
Resumen
Nuestro objetivo fue determinar el efecto anticonceptivo y postcoital del extracto etanólico de las hojas de Desmodium molliculum (HBK).DC en ratas hembras adultas Holtzmann. Las hojas fueron recolectadas en el valle de Baños del Inca, departamento de Cajamarca, la cual es utilizada como diurética, depurativa de la sangre, antihemorrágica, antiinflamatoria de las vías urinarias, hígado y riñones. Se utilizaron 80 ratas hembras fueron seleccionadas aleatoriamente de acuerdo a los criterios de inclusión, y divididas en 2 grandes grupos, cada uno conformada por 5 subgrupos de 8 ratas hembras y ratas machos para el emparejamiento (1 macho: 2 hembras). Grupo 1: 40 ratas para la evaluación del efecto anticonceptivo del extracto de Desmodium molliculum a una solución $100 \mathrm{mg} / \mathrm{ml}$ vía oral en dosis $200 \mathrm{mg} / \mathrm{kg}, 600 \mathrm{mg} / \mathrm{kg}$ y $1000 \mathrm{mg} / \mathrm{kg}$. Se utilizaron dos grupos como controles suero fisiológico y Medroxiprogesterona en dosis 15mg/kg; Grupo 2: 40 ratas para la evaluación del efecto postcoital del extracto Desmodium molliculum a una solución de $100 \mathrm{mg} / \mathrm{ml}$ vía oral a dosis $200 \mathrm{mg} / \mathrm{kg}, 600 \mathrm{mg} / \mathrm{kg}$ y $1000 \mathrm{mg} / \mathrm{kg}$. Se utilizaron dos grupos control suero fisiológico y Levonorgestrel a dosis de $50 \mathrm{ug} / \mathrm{kg}$. El efecto anticonceptivo se evaluó mediante la cuantificación de los indicadores gravidez, número de implantaciones y número de fetos; el efecto post-coital se evaluó los indicadores gravidez, número de implantaciones, número de fetos vivos y número de fetos muertos. Del escreening fitoquímico se observa que las muestras en estudio, presentan una considerable cantidad de metabolitos secundarios, destacándose cantidades apreciables de flavonoides, compuestos fenólicos, esteroides, alcaloides y taninos estuvieron en mayor cantidad en el extracto etanólico. De las evaluaciones las ratas que recibieron la solución alcohólica a dosis de $200 \mathrm{mg} / \mathrm{kg} .600 \mathrm{mg} / \mathrm{kg}, 1000 \mathrm{mg} / \mathrm{kg}$ disminuyeron la gravidez, el número fetos e implantaciones en la actividad anticonceptiva y poscoital. Se realizaron análisis descriptivos y pruebas estadísticas de significancia ANOVA y Tukey $(p<0.05)$. Concluyendo que en condiciones experimentales los resultados encontrados demuestran que el extracto etanólico de Desmodium molliculum tiene efecto anticonceptivo y postcoital en ratas hembras Holtzmann.
\end{abstract}

Descriptores: Anticonceptivo, Postcoital, Desmodium molliculum, Plantas Anticonceptiva.

\begin{abstract}
Our objective was to determine the effect of postcoital contraception and ethanol extract of the leaves of Desmodium molliculum (HBK). DC Holtzmann in adult female rats. The leaves were collected in the valley of Baños del Inca, Cajamarca department, which is used as a diuretic, purifying the blood, prevent bleeding, inflammatory urinary tract, liver and kidneys. We used 80 female rats were randomly selected according to the inclusion criteria, and divided into 2 groups, each consisting of 5 subgroups of 8 male rats and female rats for pairing (1 male: 2 females).
\end{abstract}


Group 1: 40 rats to evaluate the contraceptive effect of the extract of Desmodium molliculum a $100 \mathrm{mg} / \mathrm{ml}$ solution orally at $200 \mathrm{mg} / \mathrm{kg}, 600 \mathrm{mg} / \mathrm{kg}$ and $1000 \mathrm{mg} / \mathrm{kg}$. Two groups as saline controls and $15 \mathrm{mg} / \mathrm{kg}$ dose medroxyprogesterone.

Group 2: 40 rats to evaluate the effect of post-coital Desmodium molliculum extract a $100 \mathrm{mg} / \mathrm{ml}$ solution orally at $200 \mathrm{mg} / \mathrm{kg}$ dose, $600 \mathrm{mg} / \mathrm{kg}$ and $1000 \mathrm{mg} / \mathrm{kg}$. Two control groups were used and Levonorgestrel saline at doses of $50 \mathrm{ug} / \mathrm{kg}$. The contraceptive effect was assessed by quantifying indicators pregnancy, number of locations and number of fetuses; the effect was evaluated post-coital pregnancy indicators, number of implantations, number of live fetuses and dead fetuses number. Phytochemical screening shows that the samples under study show a considerable amount of secondary metabolites, highlighting significant amounts of flavonoids, phenolic compounds, steroids, alkaloids and tannins were in greater amounts in the ethanol extract. Evaluation of rats receiving a dose of alcoholic solution $200 \mathrm{mg} / \mathrm{kg} .600 \mathrm{mg} / \mathrm{kg}, 1000 \mathrm{mg} / \mathrm{kg}$ decreased pregnancy, fetuses and the number deployments and postcoital contraceptive activity. Descriptive analyzes were performed and statistical tests of significance ANOVA and Tukey $(p<0.05)$. Concluding that under experimental conditions the results show that the ethanol extract of Desmodium molliculum effect and postcoital contraception in female rats Holtzmann.

\section{Introducción}

El control de la fertilidad constituye un problema de salud global, como la superpoblación tiene tanto impacto personal y social que es necesario para su control en el tiempo. Como sabemos el anticonceptivo completo disponible en el mercado no están a salvo, en su mayoría son de naturaleza esteroidea y tienen efectos adversos peligrosos. La atención se ha centrado en las plantas autóctonas para posible efecto anticonceptivo $[1,2]$. El conocimiento tradicional sobre el uso medicinal de las plantas debe ser evaluado bajo condiciones de laboratorio utilizando ensayos biológicos adecuados.

El control de la natalidad forma parte del sistema de planificación familiar de todas las sociedades a nivel mundial debido a que cuando está incrementada muchas veces conlleva problemas sociales, económicos y de salud a nivel familiar. Actualmente se realizan esfuerzos para llevar y desarrollar nuevos sistemas y medios para el control de la natalidad. Uno de ellos es el desarrollo de contraceptivos orales efectivos $[3,4]$. La fuente de los contraceptivos se ha desarrollado a partir de los principios activos de numerosas plantas que históricamente han sido utilizadas para reducir la fecundidad en diferentes culturas ancestrales, como es evidente en algunos libros, manuscritos y revisiones disponibles [5], y mediante la Investigación científica moderna se han confirmado los efectos de antifertilidad de dichas planta[6].

Una de las plantas que ha reportado el uso folklórico para el control de la natalidad es la Desmodium molliculum. Esta planta está ampliamente distribuida en diversas zonas de nuestro país [7], es una especie que crece en los departamentos de Huánuco, Junín, Cuzco, Ayacucho, La Libertad, Lima y Cajamarca [8. También tiene usos etnobiomédicos como diurético, antihemorrágico, depurativo sanguíneo, antiinflamatorio de las vías urinarias, hígado y riñones $[7,8]$. Sin embargo, a pesar de su potencial efecto farmacológico en el control de la natalidad, en nuestro medio no hay evidencias cientificas que corroboren el uso tradicional.Y en este sentido el trabajo es una aportación para su uso racional. Por ello, esta investigación tuvo por objetivo de obtener los principales metabolitos secundarios mediante un análisis preliminar,asi mismo para determinar el efecto anticonceptivo y postcoital del extracto etanólico de Desmodium molliculum; y comparar el efecto contraceptivo de la solución etanólica con fármacos contraceptivos progestágenos. Finalmente observar las evaluaciones histopatológicas de úteros en ratas hembras albinas holtzmann.

\section{II.Mètodo}

2.1.Recolección e identificación del Material Vegetal 
Las hojas de la planta Desmodium molliculum fueron recolectadas en el valle de Los baños del Inca del Departamento de Cajamarca. La identificación taxonómica de la planta fue realizada en el Museo de Historia Natural de la Universidad Nacional Mayor de San Marcos, según constancia No 061-USM-2008.

División: Magnoliophyta

Clase: Magnoliopsida

Subclase: Rosidae

Orden: Fabales

Familia: Fabaceae

Género: Desmodium molliculum (HBK) DC

Nombre Común: "Manayupa"

\subsection{Preparación del extracto etanólico}

Para la preparación del extracto etanólico de de las hojas de Desmodium molliculum [10] recolectadas, fueron llevadas a una cámara de secado con menos de $40^{\circ} \mathrm{C}$ durante un periodo de 72 horas, luego, fueron trituradas en un molino eléctrico de cuchillas, hasta obtener un polvo fino, macerándose con alcohol etílico $96^{\circ}$. Al término de los 5 días se filtró y se llevó a estufa a $40^{\circ} \mathrm{C}$ en placas grandes, para la desecación respectiva durante 48 horas para llevarlo a extracto seco.

\subsection{Screening Fitoquímico}

El anàlisis fitoquímico [11] para los diferentes constituyentes activos de la planta en extracto seco, se realizó reacciones de identificación y coloración para determinar la presencia o ausencia de metabolitos activos haciendo uso de reactivos específicos realizándose $5 \mathrm{mg}$ de extracto problema con 5 gotas de reactivo y ensayos para detectar alcaloides, compuestos fenólicos,taninos,aminoácidos,esteroides, saponinas basado en la aplicación de pruebas de coloración y precipitación.

\subsection{Manejo de los animales de experimentación}

Los animales durante la investigación fueron manejados siguiendo las normas para el manejo ético de animales de experimentación [12]. Se emplearon 80 ratas hembras (2-4 meses de edad) y 40 ratas machos de la cepa Holtzmann en edad reproductiva. Las ratas hembras tuvieron un peso promedio entre 180 a $200 \mathrm{~g}$ y los machos $280 \mathrm{a}$
300 g. Estos animales fueron obtenidos del Instituto Nacional de Salud Centro Nacional de Alimentación y Nutrición.

La parte experimental fue realizada en el Bioterio de la Facultad de Medicina de la UNMSM. En dicho ambiente se mantuvieron las condiciones de temperatura $\left(22^{\circ} \mathrm{C}\right)$, ciclo de luz (12 horas de luz y 12 de oscuridad), con dieta balanceada y agua libre.

\subsection{Estudio Farmacológico}

\subsubsection{Actividad Anticonceptiva}

En la evaluación de la actividad anticonceptiva se realizó siguiendo la técnica descrita por Nwafor et al.2001 $\left({ }^{13}\right)$. Fueron 80 ratas hembras y 40 ratas machos de raza cepa Holtzmann del Instituto Nacional de Salud del Perú, los cuales fueron divididos en 2 grupos de 40 ratas, cada uno con 5 subgrupos de 08 ratas hembras por cada subgrupo.

Antes de inicio la distribución de las ratas hembras tenían un ciclo estrogénico regular, el cual fue confirmado por análisis de residuos vaginales. La secreción vaginal se recogió con una pipeta de vidrio en solución salina normal $(\mathrm{NaCl} 0,9 \%)$ se introdujo en la vagina de cada rata, de forma superficial, utilizándose portaobjetos de vidrio. El material sin teñido, ni manchas se observo en un microscopio de luz a 10X y 40X de lentes objetivo. Los tipos de células epiteliales se reconocen la cornificación, un frotis de estro se compone de células cornificadas anucleadas y los pequeños redondos son los leucocitos. La proporción entre estas células fueron usadas para determinar las fases del estro de acuerdo a Long \& Evans 1922 ${ }^{(14)}$.Las ratas que se encuentran en ciclo estral proestro fueron seleccionadas, con ratas machos de fertilidad probada, en la proporción 2:1 y examinada al día siguiente por la mañana para la prueba de copulación. Las ratas que exhiben grupos grueso de espermatozoides en su frotis vaginal fueron separados y ese día fue designado como día 1 de la gestación durante 1 semana se le administró el extracto etanólico a los respectivos grupos de experimentación, en dosis de $200 \mathrm{mg} / \mathrm{kg}$, $600 \mathrm{mg} / \mathrm{kg}, 1000 \mathrm{mg} / \mathrm{kg}$, el grupo control recibió solución salina fisiológica $5 \mathrm{ml} / \mathrm{kg}$ por vía oral a través de la sonda intragástrica a sus respectivos 
grupos, mientras que la medroxiprogesterona $15 \mathrm{mg} / \mathrm{kg}$ se administró vía intramuscular. Durante este período se evitó la presencia de los machos. El día 7 se adicionó una rata macho por cada dos ratas hembras en cada grupo hasta el día 15.

Luego, a los 10 días del embarazo las ratas fueron anestesiadas con pentobarbital solución $30 \mathrm{mg} / \mathrm{ml}$ a dosis de $1 \mathrm{~m} / 100 \mathrm{~g}$ y laparatomizadas para evaluar la contracepción, observando el número de fetos en el útero.

\subsubsection{Actividad Poscoital}

En la evaluación de la actividad poscoital se realizó el experimento siguiendo la técnica descrita por Shirishailappa et al 2003 [15], y Muller et al 2003 [17].

Las ratas con comprobada fertilidad y ciclo estrogénico regular fueron enjauladas por una noche con machos de probada fertilidad en una proporción de 2: 1 (hembra: macho). A la mañana siguiente las ratas hembras fueron examinadas por evidencia de copulación. Los animales que mostraban grupos gruesos de espermatozoides de vagina fueron separadas y ese día se designó como el día uno del embarazo. Luego se separó al macho. A continuación se administró la solución respectiva a cada subgrupo $200 \mathrm{mg} / \mathrm{kg}, 600 \mathrm{mg} / \mathrm{kg}$, $1000 \mathrm{mg} / \mathrm{kg}$ y Levonorgestrel $50 \mathrm{ug} / \mathrm{kg}$ por vía oral a través de la sonda intragástrica desde el día 1 al 7 de embarazo.

A los 7 días del embarazo las ratas fueron anestesiadas con pentobarbital y laparatomizadas para el registro del indicador macroscópico preñez y los indicadores microscópicos número de implantes, número de fetos vivos y número de fetos muertos.

\subsubsection{Evaluación Histopatología}

La evaluación histopatología según Devi et al 2007 [17], se remitieron las muestras uterinas que fueron extirpados y lavados suavemente con solución salina, conservadas en formol al $10 \%$ tamponados por 7 días para posterior estudio histopatológico al servicio de anatomía patológica del Hospital Loayza, donde se realizaron los cortes histológicos quedando la lectura de láminas para posterior interpretación, mientras que los observadores de los cortes histológicos, ciegos al tipo de muestra para evitar la subjetividad.

\subsubsection{Análisis Estadístico}

La eficacia anticonceptiva y poscoital fue analizada mediante la prueba ANOVA y Tukey $(p<0.05)$. El análisis estadístico fue realizado utilizando el programa de software SPSS versión16 para Windows 2009.

\section{Resultados}

Se obtuvo un extracto etanólico con aspecto de masa homogénea, consistencia blanda, color verde petróleo, libre de partículas extrañas, el rendimiento fue de $2 \%$ de la planta entera. El anàlisis fitoquímico se realizò determinaciones cualitativas que demostró que los flavonoides, compuestos fenólicos, taninos, esteroides, saponinas, alcaloides y carbohidratos, estuvieron en mayor cantidad en la fracción etanòlica (Tabla 1).

En la Fig.1 se observa, que existe asociación entre el efecto anticonceptivo del extracto de Desmodium molliculum y el indicador Gravidez. También se gráfica que no se observó una relación entre el aumento de la dosis del extracto investigado y el indicador Gravidez.

Tabla 1: Screening fitoquimico del extracto etanòlico de las hojas de Desmodium molliculum

\begin{tabular}{|l|l|l|}
\hline Reactivo & $\begin{array}{l}\text { Metabolitos primarios y } \\
\text { secundarios }\end{array}$ & Resultado \\
\hline $\mathrm{AlCl}_{3}$ & Flavonoides & +++ \\
\hline $\mathrm{FeCl}_{3}$ & Compuestos fenólicos & +++ \\
\hline Shinoda & Flavonoides & +++ \\
\hline Gelatina-sal 1\% & Taninos & +++ \\
\hline Liebermann - Bouchard & Esteroides & +++ \\
\hline Salkowski & Esteroides & +++ \\
\hline Rosenheim & Esteroides & +++ \\
\hline Indice afrosimétrico & Saponinas esteroidales & +++ \\
\hline Dragendorff & Alcaloides & +++ \\
\hline Mayer & Alcaloides & +++ \\
\hline Popoff & Alcaloides & +++ \\
\hline Wagner & Alcaloides & +++ \\
\hline Fehling A,B & Azúcares reductores & - \\
\hline Ninhidrina & Aminoácidos & - \\
\hline Molish & Carbohidratos & +++ \\
\hline
\end{tabular}

Leyenda: (-) No detectable, (++) Moderado, (+++) Abundante 
En la Fig. 2 se observa, de manera descriptiva, el efecto anticonceptivo del extracto de Desmodium molliculum y los indicadores microscópicos para la evaluación del efecto anticonceptivo: número de implantaciones y número de fetos. Se puede notar claramente que el extracto tuvo un efecto marcado en relación al suero fisiológico y menor que la Medroxiprogesterona según la concentración del extracto para ambos indicadores, siendo este hallazgo estadísticamente significativo $(p<0,05)$.

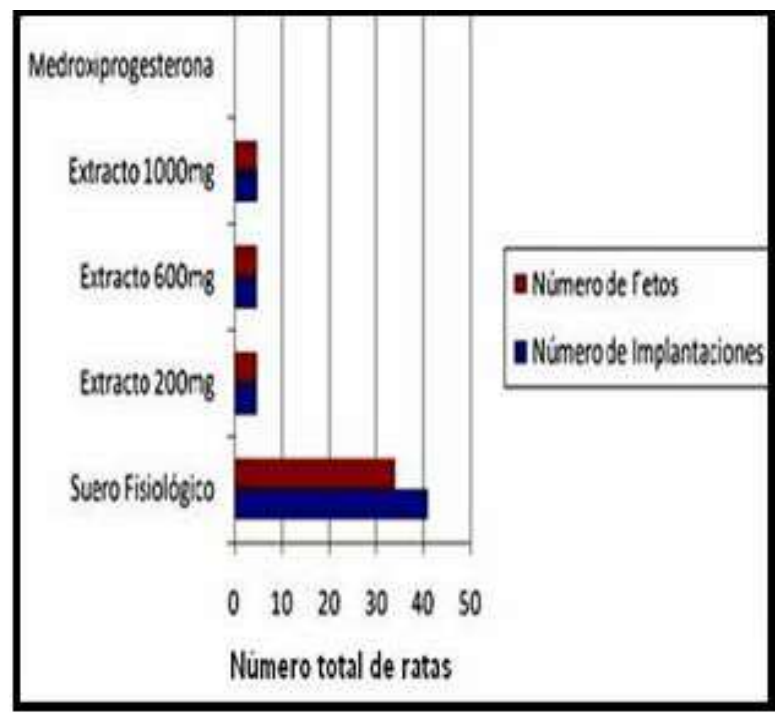

Fig. 2: Efecto Anticonceptivo según Indicadores Microscópicos

También se observa mayor relación entre el aumento de la dosis del extracto investigado y los indicadores microscópicos, aunque esta fue estadísticamente significativo para los subgrupos extracto y medroxiprogesterona frente al subgrupo suero fisiológico $(p<0,05)$; mientras que no hubo diferencia estadística significativa entre los subgrupos extracto y el subgrupo medroxiprogesterona $(p<0,05)$

En la Fig. 4 se observa que el extracto de Desmodium molliculum disminuyó el número de fetos vivos, hallazgo que fue estadísticamente significativo para todos los subgrupos extracto en relación al subgrupo suero fisiológico, así como entre los subgrupos $200 \mathrm{mg} / \mathrm{kg}$ y $600 \mathrm{mg} / \mathrm{kg}$ con el subgrupo levonorgestrel, en tanto que no hubo diferencia estadística significativa entre los resultados para los subgrupos levonorgestrel y extracto $1000 \mathrm{mg} / \mathrm{kg} \quad(p<0,05)$. Asimismo, no se encontró diferencia estadística significativa entre los subgrupos extracto.

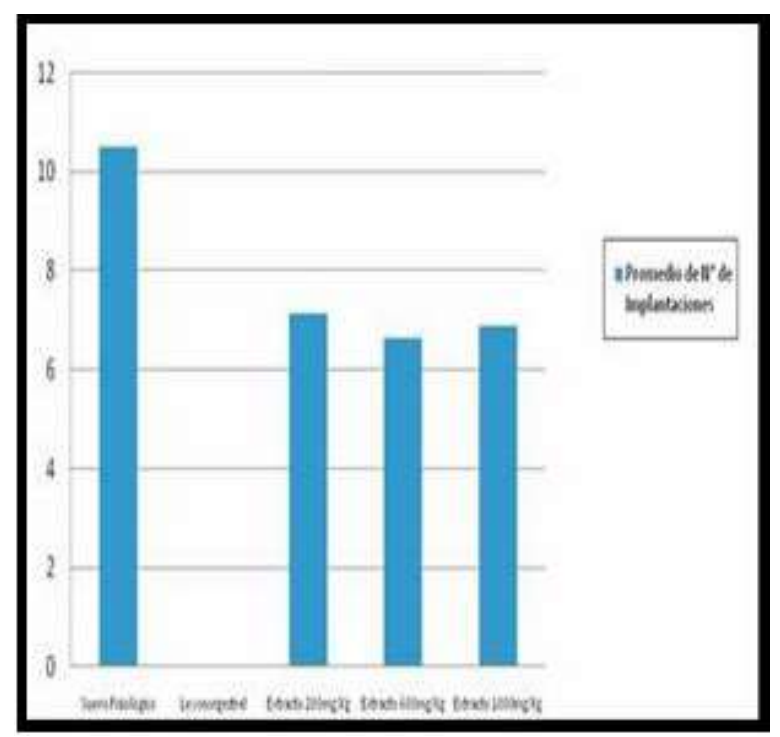

Fig. 3: Efecto Postcoital del Extracto Etanólico de Desmodium molliculum (HBK) DC. "Manayupa" en ratas hembras Holtzmann

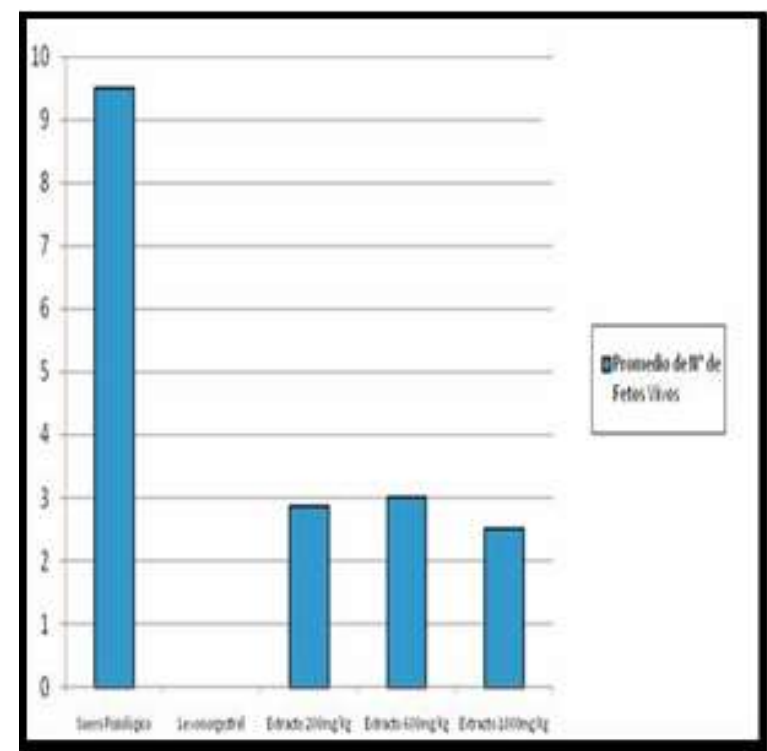

Fig. 4: Efecto Postcoital del Extracto Etanólico de Desmodium molliculum (HBK) DC. "Manayupa" en ratas hembras adultas Holtzmann

En cuanto a los indicadores microscòpicos en la Fig. 3 se observa, de manera descriptiva, el efecto Postcoital del extracto de Desmodium molliculum y el indicador número de implantaciones, en donde se observa que el extracto de Desmodium 
molliculum disminuyó el número de implantaciones, aunque este hallazgo no fue estadísticamente significativo en relación al subgrupo suero fisiológico $(p<0,05)$. Asimismo, no se encontró diferencia estadística significativa entre los subgrupos extracto.

En relación, al indicador número de fetos muertos, en la Fig. 5 se observa que en los subgrupos extracto de Desmodium molliculum un mayor número de fetos muertos en comparación a los subgrupos suero fisiológico y levonorgestrel, siendo estos hallazgos estadísticamente significativos $(p<0,05)$. Asimismo, no se encontró diferencia estadística significativa entre los subgrupos extracto.

Los estudios anatomopatològicos y el análisis macroscópicos de las muestras, comunes a todas las muestras uterinas se observa con signos inflamatorios, edematizadas, hipertrofiados y presencia de lesiones de buena adherencia, color y dimensiones. Las muestras examinadas fueron uniformes de aproximadamente $3 \times 2 \times 2 \mathrm{~mm}$ para la evaluación histológica (Figs. 6 al 12).

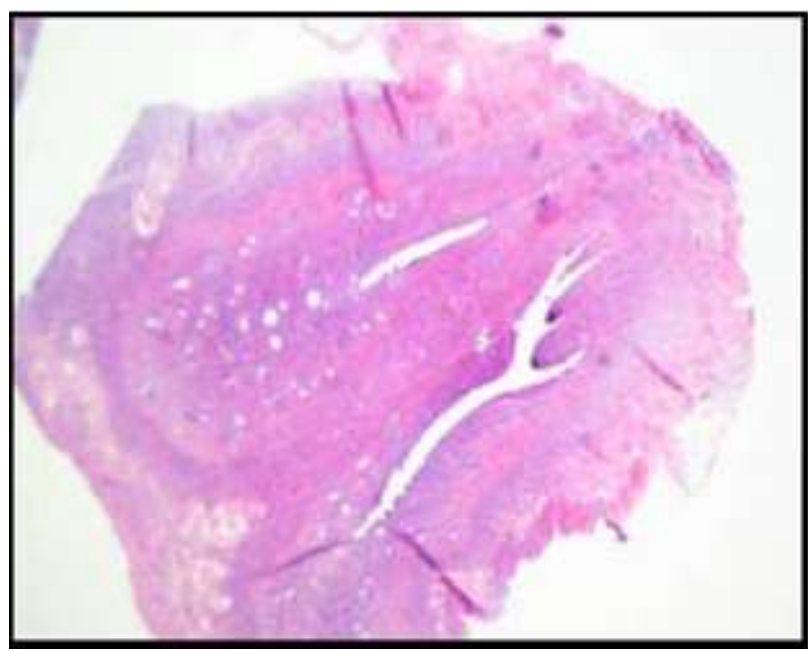

Fig. 6: Corte Histológico del Útero de rata tratado con extracto de Desmodium molliculum $200 \mathrm{mg} / \mathrm{kg}$. Anticoncepción. Hiperplasia endometrial y formación decidual (Coloración H.E. Aumento 200x) $n=8$

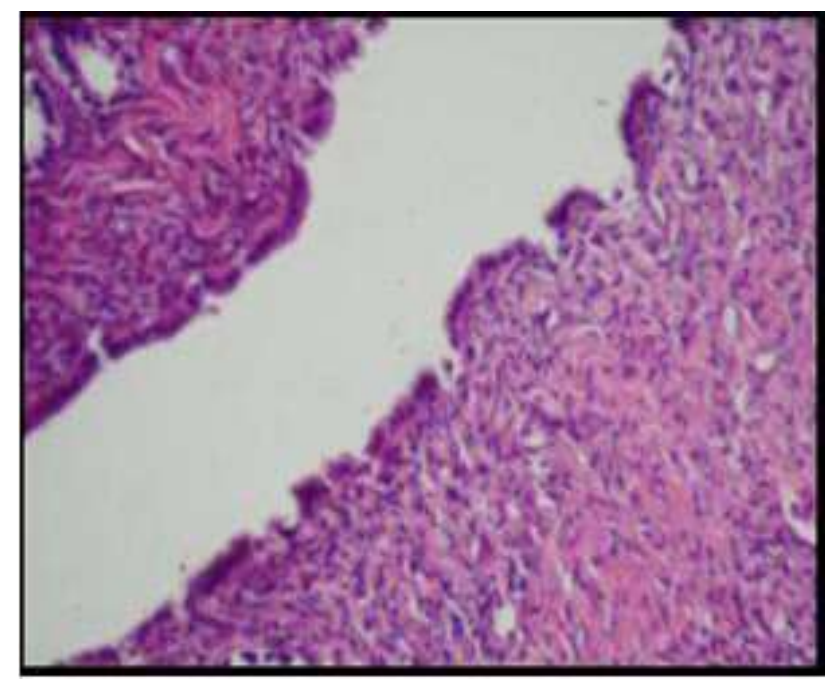

Fig. 7: Corte Histológico del Útero de rata tratado con extracto de Desmodium molliculum $600 \mathrm{mg} / \mathrm{kg}$. Anticoncepción. Lumen uterino ligeramente distendido, supresión de glándulas endometriales. No grávida (Coloración H.E. Aumento 200x) $n=8$

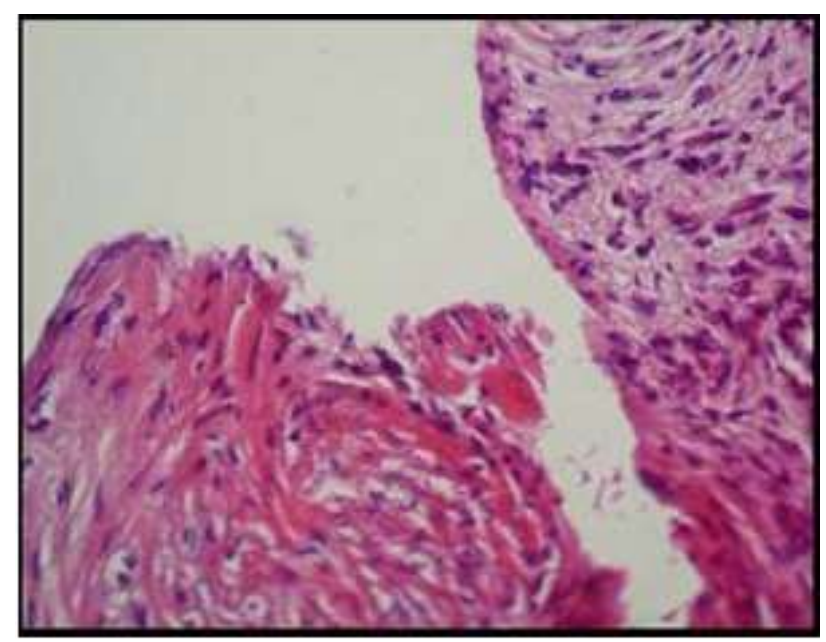

Fig. 8: Corte Histológico del Útero de rata tratado con extracto de Desmodium molliculum $1 \mathrm{gr} / \mathrm{kg}$. Anticoncepción. Signo de alteración en la estructura endometrial alta pérdida de Lumen endometrial Útero gestante descamado, No grávida (Coloración H.E. Aumento 200x) $n=8$ 


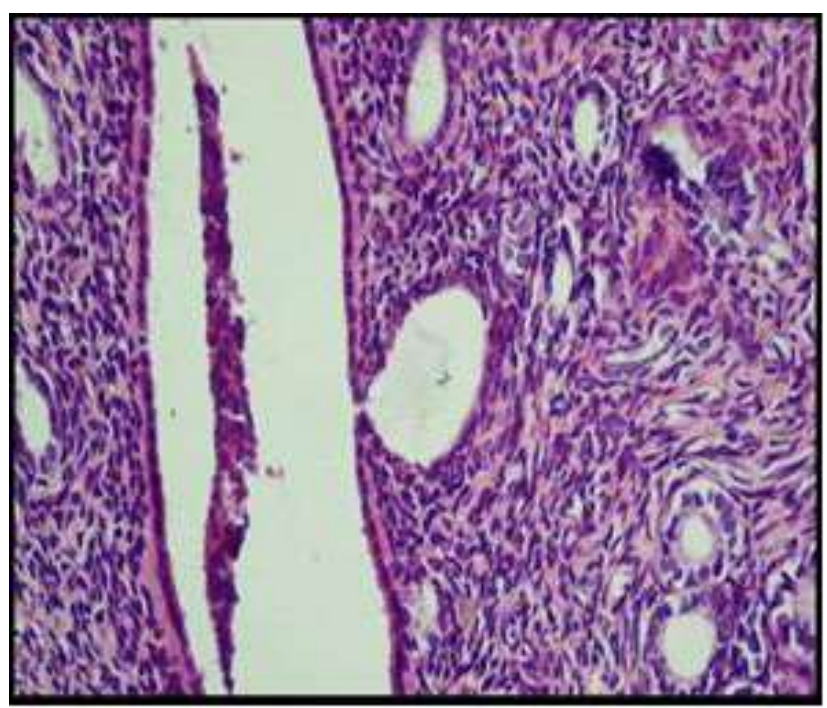

Fig. 9: Corte Histológico del Útero de rata tratado com medroxiprogesterona $15 \mathrm{mg} / \mathrm{kg}$. Anticoncepción. Se observa hiperplasia endometrial glandular, abundantes áreas quísticas. No gestante (Coloración H.E. Aumento 200x) $n=8$

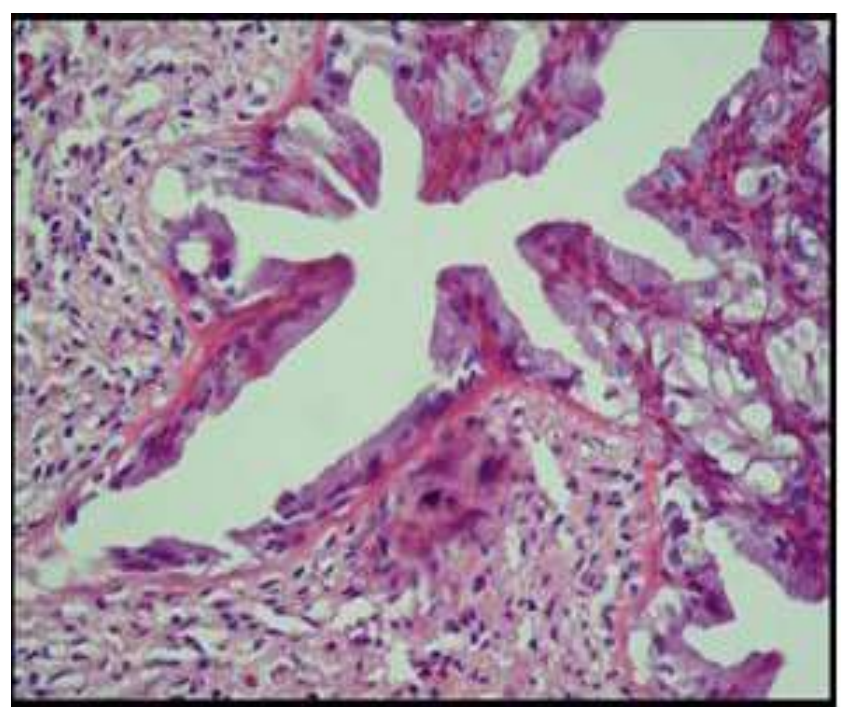

Fig. 10: Corte Histológico del Útero de rata tratada con extracto de Desmodium molliculum $200 \mathrm{mg} / \mathrm{Kg}$ Postcoital. Utero gestante, endometrio empastado e hiperplásico (Coloración H.E. Aumento 200x) $n=8$

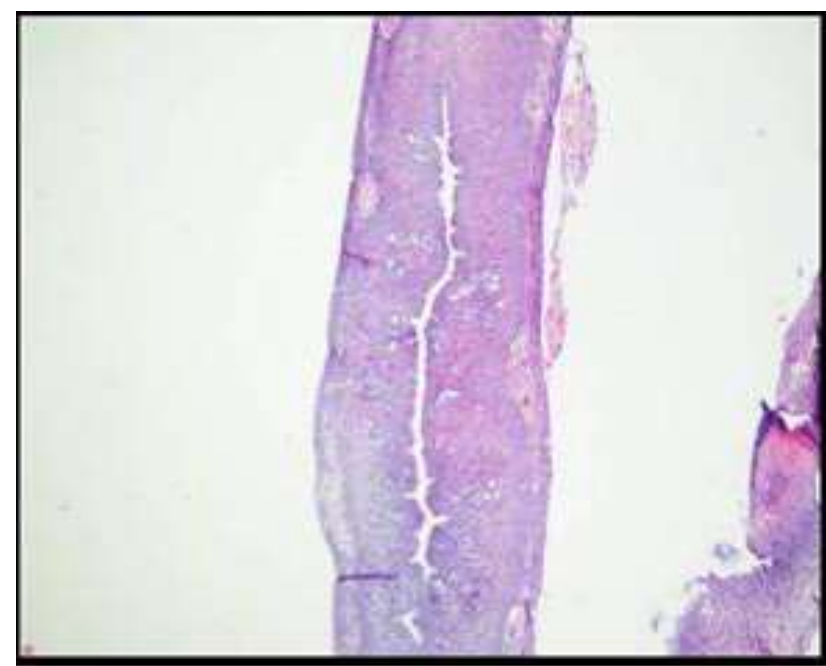

Fig. 11: Corte Histológico del Útero de rata tratado con extracto de Desmodium molliculum $1 \mathrm{gr} / \mathrm{kg}$. Post coital. Útero No gestante uniformidad endometrial, hiperplásico (Coloración H.E. Aumento 200x) $n=8$

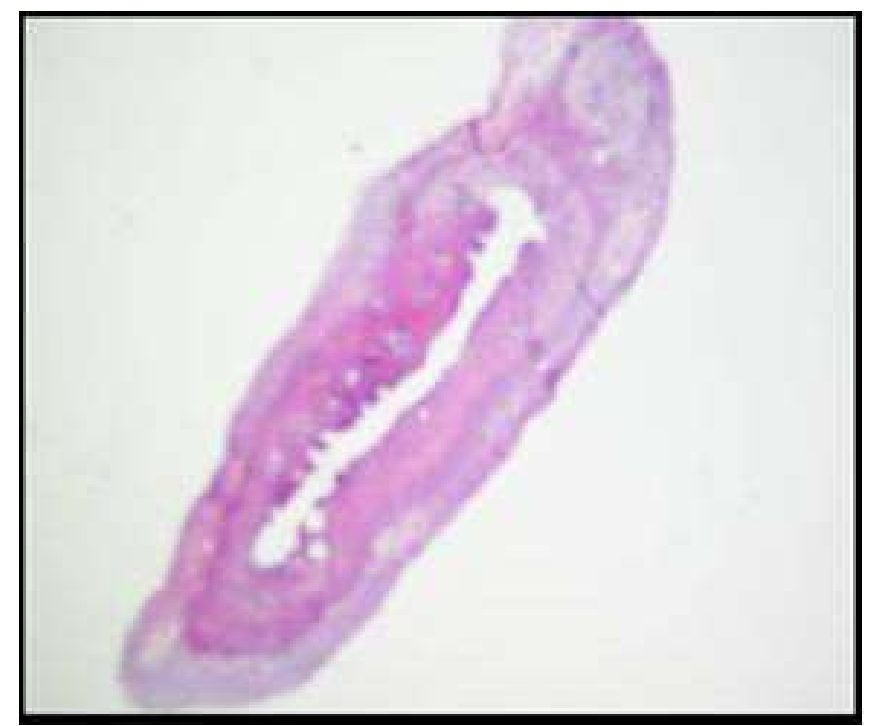

Fig. 12: Corte Histológico del Útero de rata tratado con levonogestrel $50 \mathrm{ug} / \mathrm{kg}$. Post coital. Aumento del engrosamiento endometrial ruptura uterina. (Coloración H.E. Aumento 100x) $n=8$ 


\section{Discusiòn}

Los resultados mostraron que el extracto utilizado posee efectos anticonceptivos y postcoitales (ver Figs.1al 5), en cuanto al mecanismo de acción de estos efectos se ha descrito una relación con sus componentes fitoestrogénicos activos. Según la tabla fitoquímica preliminar del extracto (tabla 1), se observa que el extracto utilizado en este estudio contiene importante presencia de alcaloides, taninos, esteroides, flavonoides, saponinas, compuestos fenólicos y carbohidratos. Falodun et al. 2001 (18) informó los efectos oxitócicos de las planta que tienen saponinas, glucósidos, esteroides, taninos y alcaloides que actuaron en úteros aislados de ratas hembras, traen como consecuencia influencias negativas sobre el ciclo estral ya que reduce el número de días/ovulación durante el proestro y estro. La mayoría de estos compuestos están presentes en Desmodium molliculum.

Es conocido que la implantación y el mantenimiento del embarazo, el equilibrio exacto de la secreción de estrógenos y progesteronas es importante. Cualquier desequilibrio en los niveles de estas hormonas puede causar antiimplantación o pueden inducir aborto. Muchos extractos de plantas con efecto antifertilidad presentan actividad estrogénico en ratas. En ratones y en humanos, los estrógenos son importantes en la implantación, ya que ellos participan en el balance estrógeno/progesterona y por esto en la receptividad uterina al embrión $(19,20)$.

En los resultados de este estudio se observó que el subgrupo de levonorgestrel tuvo un marcado efecto en los indicadores analizados, número de implantaciones y número de fetos vivos y muertos (Ver Figs. 3,4,5). El efecto del levonorgestrel (LNG), el cual se utilizó como grupo referencia, Croxatto et al, 2004 (21) expusieron a ratas hembras a dosis elevadas de levonorgestrel en varias etapas del ciclo reproductivo antes o después de la ovulación o antes o después del apareamiento. Los investigadores encontraron que el levonorgestrel inhibió total o parcialmente la ovulación, dependiendo del momento en que fue administrado así como de su dosis. Sin embargo, el fármaco no tuvo ningún efecto en la fecundación o implantación cuando se administró antes o después del apareamiento o antes de la implantación. Palomino et al, 2003 (22), indicaron en condiciones que el levonorgestrel administrado por vía oral, no altera el proceso ovulatorio, no impide la síntesis de progesteronas por el cuerpo lúteo. El LNG no modifica el patrón de expresión de los receptores de progesterona durante el proceso de la implantación.

El extracto ha demostrado ser muy significativa la actividad antiimplantación cuando se administró a partir del día 1 al 7 . El extracto fue dado durante diferentes períodos de gestación en las etapas sucesivas de la embriogénesis (23). A partir de nuestros resultados es evidente que el extracto inhibe la implantación cuando se administra en la fase cigótica (días 1-3) y en la etapa del blastocito de 3 a 5 días.

Figs. del 6 al 8 , muestran úteros de ratas tratadas con diferentes dosis del extracto Desmodium molliculum, así mismo la Fig. 9 (Medroxiprogesterona a dosis $15 \mathrm{mg} / \mathrm{kg}$ ), al realizar las comparaciones de ambas se mostró a dosis dependiente supresión y degeneración del endometrio, estroma endometrial y engrosamiento de los vasos sanguíneos del endometrio. Estos efectos son indicativos de la potencia anticonceptiva del Desmodium molliculum. Estos efectos son más probablemente debido a los desequilibrios en el nivel hormonal provocado por el elevado nivel de flavonoides, saponinas esteroidales, y otros fitoestrógenos. El grosor del endometrio varía considerablemente de acuerdo al estado hormonal del individuo (24).

Las Figs. 10 y 11, muestran úteros de ratas tratadas con extracto de Desmodium molliculum a dosis $200 \mathrm{mg} / \mathrm{kg}$ y $1 \mathrm{gr} / \mathrm{kg}$ respectivamente se comparó con la Fig. 12 de levonorgestrel a dosis $50 \mathrm{ug} / \mathrm{kg}$., se muestra cambios endometriales hiperplásicos, glándulas dañadas, degeneración epitelial/necrosis, cuando se compara con la Fig, 11, es posible que el extracto de Desmodium 
molliculum pueda interferir con el proceso de fertilización en el oviducto (Pre-implantación) de los úteros $\mathrm{o}$ un deterioro en la producción de citoquinas, factores de crecimiento y distintos tipos de molécula de adhesión, ya sea por el blastocito en desarrollo o del epitelio uterino en todo el sitio de implantación( 25,26).

\section{Conclusiónes}

1.Se ha demostrado que el extracto etanòlico de las hojas de Desmodium molliculum administrado por vìa oral evidenció efecto anticonceptivo y poscoital en ratas hembras holtzmann..

2.El efecto anticonceptivo y poscoital de Desmodium molliculum probablemente se deba principalmente a la acción de los flavonoides con actividad estrogénicas y no estrogénicas a nivel uterino.

3. El efecto anticonceptivo y postcoital del extracto etanòlico,no tuvo efecto dependiente de la dosis utilizada.

4. Los cambios histopatologicos de úteros de ratas tratadas con diferentes dosis del extracto, demostró daño, engrosamiento, supresión a nivel endometrial, indicando alteraciones en la reproducción.

\section{Agradecimiento}

El autor está agradecido a la Facultad de Medicina de la UNMSM por brindar sus instalaciones del Laboratorio de Farmacología y el Bioterio, para la realización de las actividades farmacológicas.

\section{Referencias}

[1] Shereen Cynthia D'Cruz, Selvaraju Vaithinathan, Rajamanickam Jubendradass, Premendu Prakash Mathur. Effects of plants and plant products on the testis. Asian $\mathrm{J}$ Androl 2010; 12: 468-479.

[2] Raghav Kumar Mishra, Shio Kumar Singh. Reversible antifertility effect of aqueous rhizome extract of Curcuma longa $L$. in male laboratory mice. Contracept 2009; 79(6): 479487.
[3] Maurya R, Srivastava S, Kulshreshta DK, Gupta CM. Traditional remedies for fertility regulation. Curr Med Chemistry 2004; 11: 1431-1450.

[4] Unny R, Chauhan AK, Joshi YC, Dobhal MP, Gupta RS. A review on potentiality of medicinal plants as the source of new contraceptive principles. Phytomedicine. 2003; 10: 233-260.

[5] Kong YC, Xie JX, But PP. Fertility regulating agents from traditional Chinese medicines. $J$ Ethnopharmacology 1986; 15(1): 1-44

[6] Mc Neil RT, Noronha CC, Kusemiju TO, Okanlawon AO. The antiovulatory effects of Ricinus communis Linn. Nig J Health Biomed. 2003; 2(1): 31-34.

[7] Brack A. Diccionario enciclopédico de plantas medicinales útiles en el Perú. CuzcoPerú.Centro de Estudios Regionales Andinos San Bartolomé de las Casas. PNUD. 1999.

[8] Brako L. Zarucchi JL. Catalogue of the flowerimngv plants and Gymnosperms of Perù. Monograph in Systematic Botany from the Missouri Botanical Garden.Missouiri.USA.1993.

[9] De Feo V. Medicinal and magical plants in tyhe northern Peruvian andes.Fitoterapia. 1991; 63(3): 426-427.

[10] Flores D, Villalobos E, Esteves R. Estudios preliminares del efecto del extracto alcohólico de Desmodium adscendens "Amor Seco" sobre la fecundidad en ratas hembras. Anales de la Facultad de Medicina UNMSM 2002;63:28-29.

[11] Lock O. Investigación fotoquímica; Métodos en el estudio de productos naturales. Fondo Editorial Pontificia Universidad Católica del Perú, 1994: 1-7.

[12] Sharp PE, The Laboratory Rat. USA. CRC Press. 1998. p.15-19.

[13] Nwafor PA,Okwuuasaba EK. Contraceptive and estrogenic effect of a methanol extract of Cassia nigricans leaves in experimental animals.Pharmaceutical Biology 2001, 39: 424-428.

[14] Long J. Evans $\mathrm{H}$. The oestrus cycle in the rat and is associated phenomena. Memoris University of California 1922; 6:1-148.

[15] Shirishailappa B,Aneesh R,Sankar S, Sathishkamar MN, Suresh B, Rajan S. Antifertility activity of Derris brevipes variety coriaceae.J Ethnopharmacol 2003, 84: 99104

[16] Müller AL, Llados CM, Croxatto HB. Postcoital treatment with levonorgestrel does 
not disrupt postfertilization events in the rat. Contraception 2003;67: 415-419.

[17] Devi RS, Narayan S, Vani G, Shyamala Devi CS. Gastroprotective efect of Terminalia arjuna bark on diclofenac sodium induced gastric ulcer. Chem Biol Interact. 2007;167(1):71-83

[18] Falodun,A, Nworgu Z A,, Ikponmwonsa M O. Phytochemical Components of Huntyeria umbellate (K. Schum) and Its Effect on Isolated Non-Pregnant Rat Uterus in Oestrus. J Pharm Sci. 2006, 19(3); 256-258.

[19] Rifal N, John J, Albers Paul SB.Fundamental of clinical chemistry, 5ta ed Lipids,Lipoproteins and apolipoproteins.WB Saunders Company; Philadelphia,2001: 462493.

[20] Dhar SK,Antifertility activity and hormonal prolife of Trans-Anethole in rats. Indian $\mathrm{J}$ Physiol Pharmacol 1995; 39:63-67.
[21] Croxatto H, Ortiz ME. Mecanismo de acción del Levonorgestrel en la anticoncepción de emergencia. Rev Chil Obstet Ginecol 2004; 69(2): 157-162.

[22] Palomino A, Boric A. Gabrer F, Espinoza A, Vega M, Devoto L. Efecto de Levonorgestrel como anticonceptivo de emergencia sobre receptores de progesterona, durante la ventana de implantación. Revista Cubana de Salud Pública 2003; 24 (1):38.

[23] Brige SJ, Mc Ewen BS, Wise MW. Serms and Women's Health.University School of Medicine Washigton.2000.p.1-15.

[24] Sponitz U.M. The functional morphology of the human endometrium and deciduas. Adv. Anat. Embryol. Cell Biol. 1992; 124: 1 - 99.

[25] Denker, HW. Implantantation a cell biological paradox. J Exp Zool. 1993; 266: 541-558.

[26] Haimovici F, Anderson DJ. Cytokines and growth factor in implantation. Microsc.Res Tech 1993; 25: 201-207

E-mail: eacaro_farmaceutico@yahoo.es 